\title{
XLII. On the tension of vapours near curved surfaces of their liquids
}

\author{
Geo. Francis Fitzgerald M.A. F.T.C.D.
}

To cite this article: Geo. Francis Fitzgerald M.A. F.T.C.D. (1879) XLII. On the tension of vapours near curved surfaces of their liquids, Philosophical Magazine Series 5, 8:50, 382-384, DOI: 10.1080/14786447908639699

To link to this article: http://dx.doi.org/10.1080/14786447908639699

曲 Published online: 13 May 2009.

Submit your article to this journal $\lceil\pi$

Џ Article views: 2

Q View related articles $₫$ 


\section{$\left[\begin{array}{ll}382 & ]\end{array}\right.$}

XLII. On the Tension of Vapours near Curved Surfaces of their Liquids. By Geo. Francis Fitzgerald, M.A., F.T.C.D.*

QIR W. THOMSON showed, in the Proc. Roy. Soc. of $D$ Edinburgh, Feb. 7, 1870, that the maximum tension of a vapour at the curved surface of its liquid when convex was greater, and when concave less than when flat. He deduced this as a consequence of the ascent of liquids in capillary tubes, by pointing out that the tension of vapour at the top and bottom of the column of liquid differs by the weight of a column of the vapour of that length, while it is impossible to suppose that there can be a continual distillation from the flat surface of the liquid to the curved one in the tube. He does not seem, however, to have observed how this result is connected with the ordinary theories of evaporation; and it is this connexion which I desire to point out.

Assuming, as seems very probable, that evaporation is due to the escape of molecules of the liquid, not from the surface only, but from a very small depth indeed beneath it as well, and that the chances of escape are less the longer the path of the molecule within the liquid, it is at once obvious that a molecule situated at a given depth below the surface will have a much better chance of escape if the surface be convex than if it be flat, and better still than if concave. On the other hand, one tending to enter the liquid from a given depth in the vapour has a less chance of entering a convex surface than a flat one, and still less than of entering a concave one. Hence, in order that the equality between the numbers entering and leaving the surface may be maintained (i.e. in order to prevent either evaporation or condensation), the tension of the vapour would have to be greater when in contact with a convex surface than when in contact with a flat, and still greater than when in contact with a concave surface.

If the matter be investigated mathematically, it may be treated very generally indeed if we assume that the depth from which evaporation takes place is so small compared with the radii of curvature of the surface of the liquid, that powers of the ratio of the former to the latter above the first may be neglected. This is certainly legitimate in all cases that can be observed; for the depth from which evaporation takes place must be very small indeed compared with the radii of curvature of the surfaces with which we have to deal.

* Communicated by the Auther, having been read at the Meeting of the British Association in Sheffield. 
Tension of Vapours near Curved Surfaces of their Liquids. 383

Let, then, the equation of the surface referred to its tangent plane be

$$
2 z=\frac{x^{2}}{a}+\frac{y^{2}}{b}+\ldots,
$$

where $a$ and $b$ are consequently the principal radii of curvature at the point, and the higher powers of $x$ and $y$ are neglected; for it is obvious that it is only points near the origin that are of any importance. Now $z$ is to be very small, so that $x^{2}$ and $y^{2}$ are small compared with $a$ and $b$. Let $r$ be the radius drawn from a point situated on $z$ at a very small distance $\gamma$. from the origin to any point on the surface, and let $\theta$ be the angle between this radius and the normal at the point where it meets the surface, and $\psi$ the angle between this normal and $z$. A molecule then emitted from $\gamma$ in the direction $r$ will have to travel a length $r$ within the liquid before escaping. Let then $f(r)$ represent the numbers emitted after having travelled this distance within the liquid. All that we know of $f(r)$ is that it vanishes for all values of $r$ above a very small quantity. Hence we may express the numbers emitted from this point by the double integral

$$
n=\iint \frac{f(r) \cos \theta d x d y}{r^{2} \cos \psi}
$$

and bearing in mind that $a$ and $b$ are large compared with such values of $r, x, y$, and $\gamma$ as do not make $f(r)$ to vanish, we can evidently expand this into the form

$$
n=\iint\left(\mathrm{A}_{0}+\frac{\mathrm{A}_{1}}{a}+\frac{\mathrm{B}_{1}}{b}\right) d x d y,
$$

where $A_{0}, A_{1}, B_{1}$ are functions of $x, y$, and $\gamma$. It is now to be observed that, on account of the symmetry of the equations involved in $x$ and $y$, we must have

$$
\iint \mathrm{A}_{1} d x d y=\iint \mathrm{B}_{1} d x d y
$$

and that, consequently, the numbers escaping from this point may be expressed as

$$
n=n_{0}+n_{1}\left(\frac{1}{a}+\frac{1}{b}\right)
$$

and if this be integrated for all depths $\gamma$, as $a$ and $b$ are the same for each the result must be of the same form, and may be written

$$
\mathrm{N}=\mathrm{N}_{0}+\mathrm{N}_{1}\left(\frac{1}{a}+\frac{1}{b}\right)
$$

In this $\mathrm{N}_{0}$ is the number that would be emitted if the surface were flat; and by changing the signs of $a$ and $b$ we get the 
384 Tension of Vapours near Curved Surfaces of their Liquids.

case of a surface curved in the opposite direction. From the considerations mentioned in the beginning of the paper, it is obvious that $\mathrm{N}_{1}$ must be positive when the surface is convex. In order to obtain the numbers admitted to the surface, we should have to substitute in the foregoing investigation the function corresponding to vapour for $f(r)$ that corresponds to the liquid; but the rest of the investigation is the same, it being recollected that when the surface of the liquid is convex that of the vapour is concave, and vice versâ. Hence in the case of a convex liquid surface, we may write those emitted as

and those admitted as

$$
\mathrm{N}=\mathrm{N}_{0}+\mathrm{N}_{1}\left(\frac{1}{a}+\frac{1}{b}\right)
$$

$$
\mathrm{N}^{\prime}=\mathrm{N}_{0}^{\prime}-\mathrm{N}_{1}^{\prime}\left(\frac{1}{a}+\frac{1}{b}\right) \text {; }
$$

and for equilibrium we must have

or

$$
\mathrm{N}=\mathrm{N}^{\prime} \text {, }
$$

$$
\mathrm{N}_{0}^{\prime}=\mathrm{N}_{0}+\left(\mathrm{N}_{1}+\mathrm{N}_{1}^{\prime}\right)\left(\frac{1}{a}+\frac{1}{b}\right) \text {. }
$$

In discussing this result, it is to be observed that an increase in the tension of the vapour probably produces little or no effect upon the numbers emitted, and that consequently $\mathrm{N}_{0}$ depends only upon the nature and temperature of the liquid; and it is the number that would be emitted or admitted if the surface were flat, and the tension the maximum corresponding to its state. On the other hand, the change in the numbers that would be admitted to a flat surface is proportional to the change in tension at that surface; so that changes in $\mathrm{N}_{0}^{\prime}$ are proportional to the changes.in tension of the vapour. We thus at once conclude that the maximum tension of vapour in contact with a convex surface of a liquid must be greater than that at a flat one by a quantity which varies directly as the sum of the curvatures of the surface. We know from Sir W. Thomson's investigation, that the coefficient by which this sum of curvatures is multiplied is proportional to the tension of the surface of the liquid ; and we can thus connect two apparently unrelated quantities, namely the rate of evaporation with the superficial tension. As $f(r)$ is the only unknown in the foregoing investigation, it might be possible to determine it by observing the rates of evaporation from drops of various sizes. That the tension of the vapour was connected with the sum of the curvatures might also have been suspected from the equilibrium of the surface requiring the normal pressure to vary with this same sum. 